PROCEEDINGS OF THE

AMERICAN MATHEMATICAL SOCIETY

Volume 140, Number 8, August 2012, Pages 2825-2834

S 0002-9939(2012)11154-2

Article electronically published on January 5, 2012

\title{
ON THE NONEXISTENCE OF PURELY STEPANOV ALMOST-PERIODIC SOLUTIONS OF ORDINARY DIFFERENTIAL EQUATIONS
}

\author{
JAN ANDRES AND DENIS PENNEQUIN
}

(Communicated by Yingfei Yi)

\begin{abstract}
It is shown that in uniformly convex Banach spaces, Stepanov almost-periodic functions with Stepanov almost-periodic derivatives are uniformly almost-periodic in the sense of Bohr. This in natural situations yields, jointly with the derived properties of the associated Nemytskii operators, the nonexistence of purely (i.e. nonuniformly continuous) Stepanov almostperiodic solutions of ordinary differential equations. In particular, the existence problem of such solutions, considered in a series of five papers of $\mathrm{Z}$. $\mathrm{Hu}$ and A. B. Mingarelli, is answered in a negative way.
\end{abstract}

\section{INTRODUCTION}

Some results about Stepanov almost-periodic solutions of ordinary differential equations indicate that these solutions are in fact Bohr almost-periodic (see e.g. $\mathrm{AB}, \mathrm{AP}, \mathrm{C} 2, \mathrm{C} 3,[\mathrm{Ha},[\mathrm{Pa}, \mathrm{Rd}, \mathrm{Ro})$. This especially concerns the BohrNeugebauer-type theorems and the Favard-type results, because solutions are assumed there to be bounded.

On the other hand, in a series of papers Hu1, Hu2, HM1, HM2, HM3, Z. Hu and A. B. Mingarelli posed and elaborated on "whether boundedness of solutions can imply their pure (i.e. nonuniformly continuous) Stepanov almost-periodicity." A Stepanov-like extension of their Favard-type results was, nevertheless, shown by M. Tarallo Ta to be just as apparent.

In $\mathrm{AP}$, we have presented several examples of scalar ordinary differential equations which admit bounded or unbounded purely Stepanov almost-periodic solutions, but in none of them were the right-hand sides Stepanov almost-periodic in a time variable. Moreover, we observed in $\mathrm{AP}$ that in order to have, under natural assumptions imposed on the right-hand sides (such as the Lipschitzianity in a space variable and the Stepanov almost-periodicity in a time variable), a purely Stepanov almost-periodic solution, none of its discretizations can be curiously (Stepanov) almost-periodic. At least in Euclidean spaces, such solutions must be, under the same conditions, still unbounded.

Received by the editors July 1, 2010 and, in revised form, March 21, 2011.

2010 Mathematics Subject Classification. Primary 34C27; Secondary 34C15, 34G20.

Key words and phrases. Stepanov almost-periodic solutions, Nemytskii operators, ordinary differential equations, nonexistence results.

The first author was supported by the Council of Czech Government (MSM 6198959214).

The second author was supported by ANR ANAR.

(C)2012 American Mathematical Society Reverts to public domain 28 years from publication 
In the present paper, we will show that also in (not necessarily finite-dimensional) uniformly convex Banach spaces (e.g. in Hilbert spaces) such solutions must be oppositely bounded and subsequently uniformly almost-periodic.

\section{Stepanov space $\mathrm{S}_{\mathrm{ap}}^{\infty}$ AND some FURTher PRELIMINARIES}

Let $E$ be a Banach space endowed with the norm $|\cdot|_{E}$. Let us recall (cf. ABG], Be, [C1, Le, [LZ]) that a function $f \in \mathrm{L}_{\mathrm{loc}}^{p}(\mathbb{R}, E), p \geq 1$, is said to be almostperiodic (a.p.) in the sense of Stepanov $\left(\mathrm{S}_{\mathrm{ap}}^{p}\right)$ if, for every $\varepsilon>0$, there corresponds a relatively dense set $\{\tau\}_{\varepsilon}$ of $\varepsilon$-Stepanov almost-periods such that

$$
\sup _{x \in \mathbb{R}}\left[\int_{x}^{x+1}|f(t+\tau)-f(t)|_{E}^{p} d t\right]^{\frac{1}{p}}<\varepsilon, \quad \text { for all } \tau \in\{\tau\}_{\varepsilon},
$$

where the integral is understood in a Bochner sense (see e.g. [PK]).

The Banach space of Stepanov almost-periodic (for short $\mathrm{S}_{\text {ap }}^{p}$ ) functions $f \in$ $\mathrm{L}_{\text {loc }}^{p}(\mathbb{R}, E), p \geq 1$, endowed with the norm

$$
\|f\|_{\mathrm{S}^{p}}:=\sup _{x \in \mathbb{R}}\left[\int_{x}^{x+1}|f(t)|_{E}^{p} d t\right]^{\frac{1}{p}},
$$

will be denoted by $\mathrm{S}_{\text {ap }}^{p}(\mathbb{R}, E)$. For $p=1$, we shall simply write $\mathrm{S}_{\text {ap }}(\mathbb{R}, E)$ and speak about $\mathrm{S}_{\mathrm{ap}}$-functions.

Since uniformly (Bohr) almost-periodic functions can be characterized, according to the Bochner theorem (see e.g. [Be, C1], LZ]), as entirely uniformly continuous $\mathrm{S}_{\mathrm{ap}}^{p}$-functions, $p \geq 1$, we can use here this characterization as their definition. By purely $\mathrm{S}_{\mathrm{ap}}^{p}$-functions, we shall therefore mean those which are not uniformly continuous.

An important role will be played by the Bohl-Bohr-Amerio theorem (see e.g. [LZ, p. 80]), which we state here in the form of the following lemma.

Lemma 2.1. In uniformly convex spaces, the boundedness of an indefinite integral of a Stepanov almost-periodic function implies its Bohr almost-periodicity.

It is well known that a linear combination of $\mathrm{S}_{\mathrm{ap}}^{p}$-functions is, for any $p \geq 1$, an $\mathrm{S}_{\mathrm{ap}}^{p}$-function. On the other hand, even for $p=1$, the product of two $\mathrm{S}_{\mathrm{ap}}^{p}$-functions need not be $\mathrm{S}_{\mathrm{ap}}^{p}$. Therefore, the properties of products will first be derived in the next section. In order for the product to be an $\mathrm{S}_{\mathrm{ap}}^{p}$-function, we need to recall the pair class $(p=1)$ of $\mathrm{S}_{\text {ap }}^{\infty}$-functions.

For more properties and details, we recommend the monographs $[\mathrm{ABG},[\mathrm{Be}$, C1], Le, and [L].

Hence, let us denote by $\chi_{A}$ the indicator of $A \subset E$ :

$$
\chi_{A}(x)= \begin{cases}1, & \text { for } x \in A, \\ 0, & \text { for } x \notin A,\end{cases}
$$

and let us consider the space $\mathrm{L}_{\mathrm{loc}}^{\infty}(\mathbb{R}, E)$ of functions $f$ s.t.

$$
\sup _{x \in \mathbb{R}}\left\|f \chi_{[x, x+1]}\right\|_{\infty}<+\infty
$$

(this means to take $p=\infty$ in standard Stepanov spaces). In fact, this space becomes $\mathrm{L}^{\infty}(\mathbb{R}, E)$, because

$$
\sup _{x \in \mathbb{R}}\left\|f \chi_{[x, x+1]}\right\|_{\infty}=\|f\|_{\infty}
$$


Indeed, the inclusion related to the inequality $\leq$ is obvious, For the reverse inequality, for any $\varepsilon>0$, we can find a set $A$ with a positive measure s.t. $|f|_{E} \geq\|f\|_{\infty}-\varepsilon$, on $A$. Thus, there exists $x \in \mathbb{R}$ s.t. $A \cap[x, x+1]$ has a positive measure and $|f|_{E} \geq\|f\|_{\infty}-\varepsilon$, on $A \cap[x, x+1]$, by which $\left\|f \chi_{[x, x+1]}\right\|_{\infty} \geq\|f\|_{\infty}-\varepsilon$.

Let us still denote by $\mathrm{S}_{\mathrm{ap}}^{\infty}(\mathbb{R}, E)$ the space of functions $f \in \mathrm{L}^{\infty}(\mathbb{R}, E)$ s.t., for any $\varepsilon>0$, there exists a relatively dense (shortly, r.d.) set $\left\{\tau_{\varepsilon}\right\}_{\varepsilon}$ s.t., for any $\tau \in\left\{\tau_{\varepsilon}\right\}_{\varepsilon}$ :

$$
\|f(.+\tau)-f(.)\|_{\infty} \leq \varepsilon .
$$

This space contains, in particular, the space of Bohr a.p. functions, but also some more general functions. For instance, any measurable essentially bounded periodic function belongs to $\mathrm{S}_{\mathrm{ap}}^{\infty}(\mathbb{R}, E)$.

Remark 2.2. We can easily check that for any measurable function $f$, we have

$$
\lim _{p \rightarrow \infty}\|f\|_{S_{\text {ap }}^{p}}=\|f\|_{\infty} .
$$

Let us prove this, provided $\|f\|_{\infty}$ is finite. The proof of the infinite case is almost the same. We know that $\|f\|_{S_{\mathrm{ap}}^{p}} \leq\|f\|_{\infty}$. For the reverse inequality, take $\varepsilon>0$. We know that there exists $x \in \mathbb{R}$ s.t. $\left\|f \chi_{[x, x+1]}\right\|_{\infty} \geq\|f\|_{\infty}-\varepsilon$. We also know that, for a sufficiently large $p,\|f\|_{\mathrm{L}^{p}([x, x+1], E)} \geq\|f\|_{\mathrm{L}^{\infty}([x, x+1], E)}-\varepsilon$. Thus, for a sufficiently large $p$, we obtain

$$
\|f\|_{S_{\text {ap }}^{p}} \geq\|f\|_{\mathrm{L}^{p}([x, x+1], E)} \geq\|f\|_{\infty}-2 \varepsilon .
$$

\section{Nemytskit's Operators in Stepanov SPACES}

Let us define the Nemytskii operator $\mathcal{N}_{f}$ built on $f: \mathbb{R} \times E \rightarrow E$ :

$$
\mathcal{N}_{f}(u):=[t \mapsto f(t, u(t))] .
$$

We begin with the particular case of products, but the product "." can have different meanings. The following lemma is an extension of the property which can be found in [Le, pp. 204-205].

Lemma 3.1. Assume that $p^{-1}+q^{-1}=r^{-1}$ with $p, q, r \in[1, \infty]$. Then:

(i) If $A \in \mathrm{S}_{\mathrm{ap}}^{p}(\mathbb{R}, \mathcal{L}(E))$ and $\beta \in \mathrm{S}_{\mathrm{ap}}^{q}(\mathbb{R}, E)$, then $A \cdot \beta \in \mathrm{S}_{\mathrm{ap}}^{r}(\mathbb{R}, E)$, where $\mathcal{L}(E)$ stands for the Banach space of all linear bounded transformations $L: E \rightarrow E$ endowed with the sup-norm.

(ii) If $\alpha \in \mathrm{S}_{\mathrm{ap}}^{p}(\mathbb{R}, \mathbb{R})$ and $\beta \in \mathrm{S}_{\mathrm{ap}}^{q}(\mathbb{R}, E)$, then $\alpha \cdot \beta \in \mathrm{S}_{\mathrm{ap}}^{r}(\mathbb{R}, E)$.

(iii) If $A \in \mathrm{S}_{\mathrm{ap}}^{p}(\mathbb{R}, \mathcal{L}(E)) \cap \mathrm{L}^{\infty}(\mathbb{R}, \mathcal{L}(E))$ and $\beta \in \mathrm{S}_{\mathrm{ap}}^{p}(\mathbb{R}, E) \cap \mathrm{L}^{\infty}(\mathbb{R}, E)$, then $A \cdot \beta \in \mathrm{S}_{\mathrm{ap}}^{p}(\mathbb{R}, E)$.

(iv) If $\alpha \in \mathrm{S}_{\mathrm{ap}}^{p}(\mathbb{R}, \mathbb{R}) \cap \mathrm{L}^{\infty}(\mathbb{R}, \mathbb{R})$ and $\beta \in \mathrm{S}_{\mathrm{ap}}^{p}(\mathbb{R}, E) \cap \mathrm{L}^{\infty}(\mathbb{R}, E)$, then $\alpha \cdot \beta \in$ $\mathrm{S}_{\mathrm{ap}}^{p}(\mathbb{R}, E)$.

Proof. Let us show the first case. It is well known (see e.g. [Be, [C1, [Le]) that we can find a set of $\varepsilon$-almost periods common to $A$ and $\beta$. Let $\tau$ be such an $\varepsilon$-almost period. We can easily check that

$$
\begin{gathered}
|A(t+\tau) \beta(t+\tau)-A(t) \beta(t)|_{E} \\
\leq\|A(t+\tau)\|_{\mathcal{L}(E)}|\beta(t+\tau)-\beta(t)|_{E}+\|A(t+\tau)-A(t)\|_{\mathcal{L}(E)}|\beta(t)|_{E} .
\end{gathered}
$$

Let us recall that, for any positive $a, b$ :

$$
(a+b)^{r} \leq 2^{r-1}\left(a^{r}+b^{r}\right) .
$$


We obtain

$$
\begin{gathered}
|A(t+\tau) \beta(t+\tau)-A(t) \beta(t)|_{E}^{r} \\
\leq 2^{r-1}\left(\|A(t+\tau)\|_{\mathcal{L}(E)}^{r}|\beta(t+\tau)-\beta(t)|_{E}^{r}+\|A(t+\tau)-A(t)\|_{\mathcal{L}(E)}^{r}|\beta(t)|_{E}^{r}\right) .
\end{gathered}
$$

Applying the well-known Hölder inequality with $p / r$ and $q / r$ as exponents, we obtain, for any $s \in \mathbb{R}$,

$$
\begin{gathered}
\int_{s}^{s+1}|A(t+\tau) \beta(t+\tau)-A(t) \beta(t)|_{E}^{r} d t \\
\leq 2^{r-1}\left(\left(\int_{s}^{s+1}\|A(t+\tau)\|_{\mathcal{L}(E)}^{p} d t\right)^{r / p}\left(\int_{s}^{s+1}|\beta(t+\tau)-\beta(t)|_{E}^{q} d t\right)^{r / q}\right. \\
\left.+\left(\int_{s}^{s+1}\|A(t+\tau)-A(t)\|_{\mathcal{L}(E)}^{p} d t\right)^{r / p}\left(\int_{s}^{s+1}|\beta(t)|_{E}^{q} d t\right)^{r / q}\right),
\end{gathered}
$$

from which

$$
\begin{gathered}
\|(A \cdot \beta)(.+\tau)-(A \cdot \beta)(.)\|_{S_{\mathrm{ap}}^{r}} \\
\leq 2^{r-1}\left(\|A\|_{S_{\mathrm{ap}}^{p}}\|\beta(.+\tau)-\beta(.)\|_{\mathrm{S}_{\mathrm{ap}}^{q}}+\|A(.+\tau)-A(.)\|_{\mathrm{S}_{\mathrm{ap}}^{p}}\|\beta(.)\|_{\mathrm{S}_{\mathrm{ap}}^{q}}\right) \\
\leq 2^{r-1}\left(\|A\|_{\mathrm{S}_{\mathrm{ap}}^{p}}+\|\beta(.)\|_{\mathrm{S}_{\mathrm{ap}}^{q}}\right) \varepsilon .
\end{gathered}
$$

The second property can be proved by the same arguments, or can be seen as a particular case, when taking $A:=[x \mapsto \alpha(t) x]$ as a linear mapping.

The two remaining cases are even a bit simpler to prove. For instance, for the last one, this begins with

$$
\begin{gathered}
|\alpha(t+\tau) \beta(t+\tau)-\alpha(t) \beta(t)|_{E}^{p} \\
\leq 2^{p-1}\left(\|\beta\|_{\infty}^{p}|\alpha(t+\tau)-\alpha(t)|_{E}^{p}+\|\alpha\|_{\infty}^{p}|\beta(t+\tau)-\beta(t)|_{E}^{p}\right),
\end{gathered}
$$

etc.

Now, consider the situation of the autonomous Nemytskii operator $\mathcal{N}_{f}$ built on $f(t, x) \equiv f(x)$. Let us denote by $\mathcal{S}_{D}(\mathbb{R}, E)$ the space which was originally denoted by $\mathcal{S}(\mathbb{R}, E)$ in D1; this means that $\mathcal{S}_{D}(\mathbb{R}, E)$ is the set of measurable functions $f: \mathbb{R} \rightarrow E$ s.t., for any $\varepsilon, \delta>0$, the set of numbers $\tau$ satisfying

$$
\sup _{\xi \in \mathbb{R}} \mu\left(\left\{t \in[\xi, \xi+1],|f(t+\tau)-f(t)|_{E} \geq \varepsilon\right\}\right)<\delta
$$

is relatively dense, where $\mu$ stands for the Lebesgue measure. Lemma 3 and its corollary in [D1] give some conditions ensuring that the Nemytskii operator maps $\mathcal{S}_{D}(\mathbb{R}, E)$ into itself. For instance, when $f$ is continuous, then the Nemytskii operator on $f$ has this property. This notion of Stepanov almost-periodicity must be compared with the usual one, used in our paper.

For this, using Danilov's notation in [D1], consider $M_{p}^{\prime}(\mathbb{R}, E)$ as the set of measurable functions $f: \mathbb{R} \rightarrow E$ s.t. $\|f\|_{S^{p}}<\infty$ and

$$
\lim _{\delta \rightarrow 0^{+}} \sup _{\xi \in \mathbb{R}} \sup _{T \subset[\xi, \xi+1], \mu(T) \leq \delta} \int_{T}|f(t)|_{E}^{p} d t=0 .
$$


The space $S_{p}$ used in [D1] is our $\mathrm{S}_{\mathrm{ap}}^{p}$, and equation (1) on p. 1420 in [D1] says that

$$
\mathrm{S}_{\mathrm{ap}}^{p}(\mathbb{R}, E)=\mathcal{S}_{D}(\mathbb{R}, E) \cap M_{p}^{\prime}(\mathbb{R}, E) .
$$

Let us now give the following consequence of Danilov's corollary:

Lemma 3.2. If $f \in \mathrm{C}^{0}(E, E), a, b>0$ and $p, q \geq 1$ satisfy

$$
\forall x \in E,|f(x)|_{E} \leq a|x|_{E}^{p / q}+b,
$$

then, for any $g \in \mathrm{S}_{\mathrm{ap}}^{p}(\mathbb{R}, E)$, we have $f \circ g \in \mathrm{S}_{\mathrm{ap}}^{q}(\mathbb{R}, E)$.

Proof. Taking $z \in \mathrm{S}_{\mathrm{ap}}^{p}(\mathbb{R}, E)$, we know that $z \in \mathcal{S}_{D}(\mathbb{R}, E)$. Thus, $f \circ z \in \mathcal{S}_{D}(\mathbb{R}, E)$, by the corollary in [D1].

Now, let us show that $f \circ z \in M_{q}^{\prime}(\mathbb{R}, E)$. For any bounded interval $T$, we have

$$
\int_{T}|f(z(t))|_{E}^{q} d t \leq 2^{q-1} \int_{T}\left(a^{q}|z(t)|_{E}^{p}+b^{q}\right) d t \leq 2^{q-1}\left(a^{q} \int_{T}|z(t)|_{E}^{p} d t+b^{q} m\right),
$$

where $m$ is the Lebesgue measure of $T$. Since $z \in L_{l o c}^{p}(\mathbb{R}, E)$, we deduce from this inequality that $f \circ z \in L_{l o c}^{q}(\mathbb{R}, E)$. Now given any $\delta>0$, any $\xi \in \mathbb{R}$ and $T \subset[\xi, \xi+1]$ whose measure is less than $\delta$, we get

$$
\int_{T}|f(z(t))|_{E}^{q} d t \leq 2^{q-1}\left(a^{q} \int_{T}|z(t)|_{E}^{p} d t+b^{q} \delta\right) .
$$

Since $z \in M_{p}^{\prime}(\mathbb{R}, E)$, we obtain from the last inequality that $f \circ z \in M_{q}^{\prime}(\mathbb{R}, E)$. So, $f \circ z \in M_{q}^{\prime}(\mathbb{R}, E) \cap \mathcal{S}_{D}(\mathbb{R}, E)=\mathrm{S}_{\text {ap }}^{q}(\mathbb{R}, E)$.

Remark 3.3. It is not true that if $f$ is continuous and $g \in \mathrm{S}_{\mathrm{ap}}$, then $f \circ g \in \mathrm{S}_{\mathrm{ap}}$. Indeed, take for instance $g=\sum_{n=2}^{+\infty} g_{n}$, with $g_{n} 4 n$-periodic, and

$$
\left.g_{n}\right|_{[-2 n, 2 n]}:=\beta_{n}\left(1-\frac{2}{\alpha_{n}}|x-n|\right) \chi_{\left[n-\alpha_{n} / 2, n+\alpha_{n} / 2\right]},
$$

where $\alpha_{n} \in(0,1 / 2)$, and $\beta_{n}>0$ will be specified later. Since $g_{n}$ is periodic, continuous and bounded, then $g_{n} \in \mathrm{S}_{\mathrm{ap}}$. Moreover, $\left\|g_{n}\right\|_{\mathrm{S}_{\mathrm{ap}}}=2 \alpha_{n} \beta_{n}$. So, if we assume that $\sum_{n} \alpha_{n} \beta_{n}<\infty$, then $g \in \mathrm{S}_{\mathrm{ap}}$. Now, as a continuous function, let us choose $f():.=\exp ($.$) . Then:$

$$
\exp (g)=\exp \left(\sum_{n} g_{n}\right) \geq \exp \left(g_{N}\right),
$$

for all $N$. Moreover,

$$
\exp \left(g_{N}\right)=\frac{\alpha_{N}}{\beta_{N}}\left(e^{\beta_{N}}-1\right) \geq \frac{\alpha_{N} \beta_{N}^{2}}{6} .
$$

So, if we have $\alpha_{n} \beta_{n}^{2} \rightarrow \infty$ when $n \rightarrow \infty$, then $\|\exp (g)\|_{\mathrm{S}_{\text {ap }}} \geq \frac{\alpha_{n} \beta_{n}^{2}}{6} \rightarrow \infty$, which verifies that $\exp (g) \notin \mathrm{S}_{\mathrm{ap}}$, when taking, for instance, $\alpha_{n}=1 / n^{5}$ and $\beta_{n}=n^{3}$.

Furthermore, let us consider the nonautonomous Nemytskii operator $\mathcal{N}_{f}$.

Proposition 3.4. Assume that

(i) for all $t \in \mathbb{R}, f(t,.) \in L^{\infty}(E, E)$;

(ii) $[t \mapsto f(t,).] \in \mathrm{S}_{\mathrm{ap}}^{q}\left(\mathbb{R}, L^{\infty}(E, E)\right)$.

Then $\mathcal{N}_{f}:=f(t, \cdot)$ maps $\mathrm{S}_{\mathrm{ap}}^{p}(\mathbb{R}, E)$ into $\mathrm{S}_{\mathrm{ap}}^{q}(\mathbb{R}, E)$, where $p, q \geq 1$. 
Proof. From Danilov's Lemma 3 in [D1], we know that if $z \in \mathrm{S}_{\mathrm{ap}}^{p}(\mathbb{R}, E)$, then $\mathcal{N}_{f}(z) \in \mathcal{S}_{D}(\mathbb{R}, E)$. Now, taking

$$
b(t):=\sup _{x \in E}|f(t, x)|_{E},
$$

we obtain

$$
|b(t+\tau)-b(t)|_{E} \leq \sup _{x \in E}|f(t+\tau, x)-f(t, x)|_{E} \leq\|f(t+\tau, .)-f(t, .)\|_{\infty} .
$$

Thus, if $\tau$ is an $\varepsilon$-almost period for $F=[t \mapsto f(t,)$.$] , we have$

$$
\|b(.+\tau)-b(.)\|_{S_{\mathrm{ap}}^{q}} \leq\|F(.+\tau)-F(.)\|_{\mathrm{S}_{\mathrm{ap}}^{q}} \leq \varepsilon
$$

which verifies that $b \in \mathrm{S}_{\mathrm{ap}}^{q}(\mathbb{R}, E)$. This implies that $\mathcal{N}_{f}(z) \in M_{q}^{\prime}(\mathbb{R}, E)$, because

$$
\int_{T}|f(t, z(t))|_{E}^{q} d t \leq \int_{T}|b(t)|_{E}^{q} d t
$$

It is possible to combine these results to obtain more general functions mapping Stepanov spaces into themselves. For instance, let us combine Lemma 3.1(i) and Lemma 3.2 to obtain immediately the following proposition.

Proposition 3.5. Set $F(t, X):=A(t) \cdot F_{1}(X), p, q \geq 1, r \in(0, p / q]$, where

(i) $A \in \mathrm{S}^{\frac{p q}{p-q r}}(\mathbb{R}, \mathcal{L}(E))$;

(ii) $F_{1} \in C^{0}(E, E)$ and satisfies:

$$
\forall X \in E, \quad\left|F_{1}(X)\right|_{E} \leq a|X|_{E}^{r}+b,
$$

with $a, b>0$.

Then $\mathcal{N}_{F}: \mathrm{S}_{\mathrm{ap}}^{p}(\mathbb{R}, E) \rightarrow \mathrm{S}_{\mathrm{ap}}^{q}(\mathbb{R}, E)$.

Proof. From Lemma 3.2 , it follows that $\mathcal{N}_{F_{1}}: \mathrm{S}_{\text {ap }}^{p}(\mathbb{R}, E) \rightarrow \mathrm{S}^{p / r}(\mathbb{R}, E)$. Thus, taking $z \in \mathrm{S}_{\text {ap }}^{p}(\mathbb{R}, E)$, we obtain $F_{1} \circ z \in \mathrm{S}^{p / r}(\mathbb{R}, E)$. Applying still Lemma 3.1(i), we arrive at $\mathcal{N}_{F}: \mathrm{S}_{\text {ap }}^{p}(\mathbb{R}, E) \rightarrow \mathrm{S}_{\text {ap }}^{q}(\mathbb{R}, E)$.

\section{MAin PROpOSition}

Let us recall that, according to the Milman-Pettis theorem (see e.g. $[\mathrm{Br}]$ ), uniformly convex Banach spaces are reflexive. Moreover, reflexivity implies the RadonNikodým property (see e.g. $[\mathrm{PK}]$ ). Thus, the following proposition holds, for instance, in Hilbert spaces.

Proposition 4.1. Let $f: \mathbb{R} \rightarrow E$ be a locally absolutely continuous function, i.e. $f \in \mathrm{AC}_{\mathrm{loc}}(\mathbb{R}, E)$, where $E$ is a Banach space satisfying the Radon-Nikodym property. If $f$ and $f^{\prime}$ are bounded in the Stepanov norm, then $f$ is bounded in the essential sup-norm. In particular, if $f \in \mathrm{AC}_{\mathrm{loc}}(\mathbb{R}, E)$, where $E$ is a uniformly convex Banach space, is s.t. $f$ and $f^{\prime}$ are Stepanov almost-periodic, i.e. $f, f^{\prime} \in \mathrm{S}_{\mathrm{ap}}^{1}(\mathbb{R}, E)$, then $f$ is Bohr (uniformly) almost-periodic.

Proof. For the first part, since $E$ has the Radon-Nikodým property (see e.g. PK, p. 694]) and since $f \in \mathrm{AC}_{\mathrm{loc}}(\mathbb{R}, E)$, the Fréchet derivative $f^{\prime}$ exists a.e. and the fundamental calculus formula holds, where the integral should be understood in Bochner's sense (see e.g. [PK, p. 695]):

$$
f(t)-f(s)=\int_{s}^{t} f^{\prime}(u) d u .
$$


First, by denoting by $[t]$ the integer part of $t$, one can easily check that

$$
|f(t)-f([t])|_{E} \leq\left\|f^{\prime}\right\|_{S_{\mathrm{ap}}^{1}} .
$$

Indeed,

$$
|f(t)-f([t])|_{E}=\left|\int_{[t]}^{t} f^{\prime}(s) d s\right|_{E} \leq \int_{[t]}^{t}\left|f^{\prime}(s)\right|_{E} d s \leq \int_{[t]}^{[t]+1}\left|f^{\prime}(s)\right|_{E} d s \leq\left\|f^{\prime}\right\|_{S_{\mathrm{ap}}^{1}} .
$$

This, in particular, yields that

$$
\sup _{t \in \mathbb{R}}|f(t)|_{E} \leq\left\|f^{\prime}\right\|_{S_{\text {ap }}^{1}}+\sup _{n \in \mathbb{Z}}|f(n)|_{E} .
$$

At the same time, for any $n \in \mathbb{Z}$, we have

$$
f(n)=\int_{n}^{n+1} f(n) d t=\int_{n}^{n+1}(f(n)-f(t)) d t+\int_{n}^{n+1} f(t) d t .
$$

Using again (1), this implies that, for any $n \in \mathbb{Z}$,

$$
|f(n)|_{E} \leq \int_{n}^{n+1}|f(n)-f(t)|_{E} d t+\int_{n}^{n+1}|f(t)|_{E} d t \leq\left\|f^{\prime}\right\|_{S_{\mathrm{ap}}^{1}}+\|f\|_{S_{\mathrm{ap}}^{1}} .
$$

Thus, we obtain

$$
\|f\|_{\infty}=\sup _{t \in \mathbb{R}}|f(t)|_{E} \leq 2\left\|f^{\prime}\right\|_{S_{\text {ap }}^{1}}+\|f\|_{S_{\text {ap }}^{1}} .
$$

For the second part of the proposition, let us consider $f \in \mathrm{AC}_{\text {loc }}(\mathbb{R}, E)$ s.t. $f$ and $f^{\prime}$ are both Stepanov almost-periodic. We know from the first part that $f$ is essentially bounded. Therefore, as a direct consequence of Lemma 2.1. we get that $f$ is uniformly almost-periodic, as claimed.

Remark 4.2. Since we obviously have (see e.g. $[\mathrm{ABG}$ )

$$
\forall p \geq 1, \quad\|f\|_{\mathrm{S}_{\mathrm{ap}}} \leq\|f\|_{\mathrm{S}_{\mathrm{ap}}^{p}},
$$

the foregoing Proposition 4.1 is also valid for $f \in \mathrm{S}_{\mathrm{ap}}^{p}(\mathbb{R}, E)$ and $f^{\prime} \in \mathrm{S}_{\mathrm{ap}}^{q}(\mathbb{R}, E)$, with any $p, q \geq 1$.

\section{Main Results}

In order to apply Proposition 4.1 to the nonexistence of purely Stepanov almostperiodic solutions, let us start this section with an intuitively clear proposition.

Proposition 5.1. Assume that $\mathcal{N}_{F}: \mathrm{S}_{\mathrm{ap}}^{p}(\mathbb{R}, E) \rightarrow \mathrm{S}_{\mathrm{ap}}^{q}(\mathbb{R}, E)$, where $p, q \geq 1$. Then every $\mathrm{S}_{\mathrm{ap}}^{p}$-solution of the differential equation $X^{\prime}=F(t, X)$, in a uniformly convex Banach space $E$, is uniformly almost-periodic.

Proof. In such cases, the derivative of any solution $x(.) \in \mathrm{S}_{\mathrm{ap}}^{p}(\mathbb{R}, E)$ will be $\mathrm{S}_{\mathrm{ap}}^{q}$, and, in view of Remark 4.2, $x($.$) as well as x^{\prime}($.$) are \mathrm{S}_{\mathrm{ap}}$. Applying the second part of Proposition 4.1 the assertion follows.

On the basis of Proposition 5.1 and the results from foregoing sections, we can consider many cases where purely Stepanov solutions do not exist. For instance, let us formulate the main result for the differential equation:

$$
X^{\prime}=A(t) \cdot F_{1}(X)+F_{2}(t, X) .
$$


Theorem 5.2. Let $E$ be a uniformly convex Banach space. Assume that there exist constants $p, q, r$ with $p, q \geq 1, r^{-1}=p^{-1}+q^{-1}$ and $r \in\left(0, \frac{p}{q}\right]$, such that the following conditions are satisfied:

(i) $A \in \mathrm{S}_{\text {ap }}^{\frac{p q}{p-q r}}(\mathbb{R}, \mathcal{L}(E))$;

(ii) $\forall X \in E, \quad\left|F_{1}(X)\right|_{E} \leq C_{1}|X|_{E}^{r}+C_{2}$, with $C_{1}, C_{2} \geq 0$, holds for $F_{1} \in \mathrm{C}^{0}(E, E)$;

(iii) for all $t \in \mathbb{R}, F_{2}(t,.) \in \mathrm{L}^{\infty}(E, E)$;

(iv) $\left[t \mapsto F_{2}(t,).\right] \in \mathrm{S}_{\mathrm{ap}}^{q}\left(\mathbb{R}, \mathrm{L}^{\infty}(E, E)\right)$.

Then equation (2) has no purely $\mathrm{S}_{\mathrm{ap}}^{p}$-solution.

Proof. Setting $F(t, X):=A(t) \cdot F_{1}(X)+F_{2}(t, X)$, let us take $z \in \mathrm{S}_{\mathrm{ap}}^{p}(\mathbb{R}, E)$. According to Proposition 3.4 the Nemytskii operator $\mathcal{N}_{F_{2}}$ maps $S_{\text {ap }}^{p}(\mathbb{R}, E)$ into $\mathrm{S}_{\mathrm{ap}}^{q}(\mathbb{R}, E)$, i.e. $\left[t \mapsto F_{2}(t, z(t))\right] \in \mathrm{S}_{\mathrm{ap}}^{q}(\mathbb{R}, E)$. Applying Proposition [3.5, we get $\left[t \mapsto A(t) F_{1}(z(t))\right] \in \mathrm{S}_{\text {ap }}^{q}(\mathbb{R}, E)$. Thus, the Nemytskii operator $\mathcal{N}_{F}$ built on $F$ maps $\mathrm{S}_{\text {ap }}^{p}(\mathbb{R}, E)$ into $\mathrm{S}_{\mathrm{ap}}^{q}(\mathbb{R}, E)$ which, in view of Proposition [5.1] completes the proof.

Remark 5.3. One can readily check that, for $F_{2}(t, X):=P(t), P \in \mathrm{S}_{\mathrm{ap}}^{q}(\mathbb{R}, E)$ need not be essentially bounded for the same conclusion. Putting still $F_{1}(X):=X$ and taking, this time, $A \in \mathrm{S}_{\mathrm{ap}}^{\infty}(\mathbb{R}, \mathcal{L}(E)), P \in \mathrm{S}_{\mathrm{ap}}^{1}(\mathbb{R}, E)$, we have by means of Lemma 3.1(i) that $[t \mapsto A(t) z(t)+P(t)] \in \mathrm{S}_{\mathrm{ap}}^{1}(\mathbb{R}, E)$, for $z \in \mathrm{S}_{\mathrm{ap}}^{1}(\mathbb{R}, E)$. Therefore, by virtue of Proposition 5.1, the linear differential equation $X^{\prime}=A(t) X+P(t)$, where $A \in \mathrm{S}_{\mathrm{ap}}^{\infty}$ and $P \in \mathrm{S}_{\mathrm{ap}}^{1}$, has no purely $\mathrm{S}_{\mathrm{ap}}$-solution. This slightly generalizes the result of M. Tarallo [Ta].

For particular forms of the right-hand sides in (2), Theorem 5.2 can still be modified as follows.

Theorem 5.4. Let $E$ be a uniformly convex Banach space. Assume that there exist constants $\alpha>1, r>0$, such that

(i) $A \in \mathrm{S}_{\text {ap }}^{\alpha}(\mathbb{R}, \mathcal{L}(E))$;

(ii) $F_{1} \in \mathrm{C}^{0}(E, E)$ and satisfies

$$
\forall X \in E, \quad\left|F_{1}(X)\right|_{E} \leq C_{1}|X|^{r}+C_{2},
$$

with $C_{1}, C_{2} \geq 0$.

Then, for any $p>\frac{\alpha r}{\alpha-1}$, equation $(2)$, where $F_{2}(t, X) \equiv 0$, has no purely $\mathrm{S}_{\mathrm{ap}}^{p}$ solution. In particular, for $r<1-\alpha^{-1}$, there are no purely $\mathrm{S}_{\mathrm{ap}}$-solutions.

Proof. For $p>\frac{\alpha r}{\alpha-1}$, we have $\frac{1}{\alpha}+\frac{r}{p} \leq 1$, and there certainly exists $q \geq 1$ such that $q^{-1}=\alpha^{-1}+(p / r)^{-1}$.

Taking $z \in \mathrm{S}_{\mathrm{ap}}^{p}(\mathbb{R}, E)$, the Nemytskii operator $\mathcal{N}_{F_{1}}$ maps, according to Lemma 3.2 $\mathrm{S}_{\text {ap }}^{p}(\mathbb{R}, E)$ into $\mathrm{S}_{\text {ap }}^{p / r}(\mathbb{R}, E)$. Hence, still applying Lemma 3.1(i), we obtain that $\left[t \mapsto A(t) F_{1}(z(t))\right] \in \mathrm{S}_{\mathrm{ap}}^{q}(\mathbb{R}, E)$ which, in view of Proposition 5.1 completes the general part of the proof.

For the particular part of the proof, it is enough to realize that, for $p=1$, the inequality $1>\frac{\alpha r}{\alpha-1}$ can be equivalently expressed as $r<1-\alpha^{-1}$. 
Remark 5.5. Under assumptions (i) and (ii), imposed on $A$ and $F_{1}$ in Theorem 5.4, Proposition 3.5 immediately implies that, for $z \in \mathrm{S}_{\mathrm{ap}}^{p}(\mathbb{R}, E)$,

$$
\left.t \mapsto A(t) F_{1}\left(z_{1}(t)\right)\right] \in \mathrm{S}_{\mathrm{ap}}^{\frac{\alpha p}{p+\alpha r}}(\mathbb{R}, E),
$$

provided the constants $\alpha, p, r$ satisfy suitable conditions, as in Proposition 3.5. The inequality $p>\frac{\alpha r}{\alpha-1}$ then simply means that $\frac{\alpha p}{p+\alpha r}>1$ by which $\left[t \mapsto A(t) F_{1}(z(t))\right] \in$ $\mathrm{S}_{\mathrm{ap}}^{1}(\mathbb{R}, E)$. Therefore, assuming (iii) and (iv) with $q \geq 1$ in Theorem 5.2, the same conclusion holds also for equation $(2)$, where $F_{2}(t, X) \not \equiv 0$.

\section{REFERENCES}

[AB] J. Andres and A. M. Bersani, Almost-periodicity problem as a fixed-point problem for evolution inclusions. Topol. Meth. Nonlin. Anal. 18, 2 (2001), 337-349. MR1911386 (2003d:34125)

$[\mathrm{ABG}]$ J. Andres, A. M. Bersani and R. F. Grande, Hierarchy of almost-periodic function spaces. Rend. Matem. Appl. 26, 7 (2006), 121-188. MR2275292 (2008b:43010)

[AP] J. Andres and D. Pennequin, On Stepanov almost-periodic oscillations and their discretizations. J. Differ. Equations Appl., to appear.

[Be] A. S. Besicovitch, Almost Periodic Functions. Dover, New York, 1954. MR0068029 $(16: 817 \mathrm{a})$

[Br] H. Brézis, Analyse fonctionnelle, théorie et applications. Masson, Paris, 1987. MR697382 (85a:46001)

[C1] C. Corduneanu, Almost Periodic Functions. Interscience Publishers, New York, 1968; Wiley, Chelsea, New York, 1989. MR0481915 (58:2006)

[C2] C. Corduneanu, Some almost periodicity criteria for ordinary differential equations. Libertas Mathematica 3 (1983), 21-43. MR722073 (85b:34056)

[C3] C. Corduneanu, Almost periodic solutions to differential equations in abstract spaces. Rev. Roum. Math. Pures Appl. 42, 9-10 (1997), 753-758. MR1656645 (99i:34083)

[D1] L. I. Danilov, Measure-valued almost periodic functions and almost periodic selections of multivalued maps. Sb. Math. 188, 10 (1997), 1417-1438; translation from the Russian original in Mat. Sb. 188, 10 (1997), 3-24. MR1485446(99e:42016)

[Ha] A. Haraux, Asymptotic behavior of two-dimensional, quasi-autonomous, almost-periodic evolution equations. J. Diff. Eqns. 66, 5 (1987), 62-70. MR.871571 (88a:34061)

[Hu1] Z. Hu, Contributions to the Theory of Almost Periodic Differential Equations. PhD Thesis, Carleton Univ., Ottawa (Ontario, Canada), 2001.

[Hu2] Z. Hu, Boundedness and Stepanov almost periodicty of solutions. Electronic J. Diff. Equations 35 (2005), 1-7. MR2135246 (2006a:34137)

[HM1] Z. Hu and A. B. Mingarelli, On a theorem of Favard. Proc. Amer. Math. Soc. 132 (2004), 417-428. MR 2022364 (2004i:34120)

[HM2] Z. Hu and A. B. Mingarelli, Favard's theorem for almost periodic processes on Banach space. Proc. Dynam. Syst. Appl. 14 (2005), 615-631. MR2179169 (2006j:34115)

[HM3] Z. Hu and A. B. Mingarelli, Bochner's theorem and Stepanov almost periodic functions. Ann. Mat. Pura Appl. 187 (2008), 719-736. MR2413376 (2009d:34115)

[Le] B. M. Levitan, Almost-Periodic Functions. Gosudarstv. Izdat. Tehn.-Teor. Lit., Moscow, 1953 (in Russian). MR0060629 (15:700a)

[LZ] B. M. Levitan and V. V. Zhikov, Almost-Periodic Functions and Differential Equations. Cambridge Univ. Press, Cambridge, 1982. MR690064 (84g:34004)

[Pa] A. A. Pankov, Bounded solutions, almost periodic in time, of a class of nonlinear evolution equations. Mathematics of the USSR - Sbornik 49, 1 (1984), 73-86; translation from the Russian original in Mat. Sb. (N.S.) 121(163), 1 (5) (1983), 72-86. MR699739 (84i:34081)

[PK] N.S. Papageorgiou and S. Th. Kyritsi-Yiallourou, Handbook of Applied Analysis. Springer, Berlin, 2009. MR 2527754 (2010g:49001)

[Rd] L. Radová, Theorems of Bohr-Neugebauer-type for almost-periodic differential equations. Math. Slovaca 54, 2 (2004), 169-189. MR2074215 (2005d:34094) 
[Ro] A. S. Rao, On differential operators with Bohr-Neugebauer property. J. Diff. Eqns. 13 (1973), 490-494. MR0328256 (48:6598)

[Ta] M. Tarallo, A Stepanov version for Favard theory. Arch. Math. (Basel) 90 (2008), 53-59. MR2382470(2009a:34016)

Department of Mathematical Analysis, Faculty of Science, Palacký University, Tř. 17 listopadu 12, 77146 Olomouc, Czech Republic

E-mail address: andres@inf.upol.cz

Université Paris I Panthéon-Sorbonne, Centre PMF, Laboratoire SAMm, 90, Rue de Tolbiac, 75634 Paris Cedex 13, France

E-mail address: pennequi@univ-paris1.fr 